\title{
TWO NEW OLEANANE-TYPE GLYCOSIDES FROM Napoleonaea imperialis P. BEAUV RIND
}

\author{
G. I. Ndukwe*, R. E. Ekong and I. R. Jack \\ Department of Chemistry, Rivers State University, Nkpolu-Oroworukwo, Port Harcourt, Nigeria \\ *Corresponding author. E-mail address: gloria.ndukwe@ust.edu.ng \\ Mobile phone: +2348033404528
}

\begin{abstract}
Napoleonaea imperialis P. Beauv (family Lecythidaceae) commonly called Napoleon's hat is a medicinal plant found in South-Eastern Nigeria. The rind was separated from the fruit and macerated using methanol. The crude methanol extract was partitioned to yield n-hexane fraction $(7.4 \%)$, dichloromethane fraction (23.7\%) and methanol fraction (68.7\%). The dichloromethane fraction was chromatographed and further purified to afford two compounds whose structures were elucidated using ${ }^{1} \mathrm{H},{ }^{13} \mathrm{C}$ and two-dimension NMR experiments. Isolated compounds, Napoleonaside $G$ and Napoleonaside $R$, were characterized as 3-O-[D-glucopyranosyl $(1 \rightarrow 2)$-D-glucopyranosyl]21,28-diangeloyloxy-24-hydroxy-olean-11,13(18)-diene and 3-O-[D-glucopyranosyl( $1 \rightarrow 4)$-Dglucopyranosyl-2"-angeloyl]-21-angeloyloxy-24,28-dihydroxy-olean-11,13(18)-diene, respectively.
\end{abstract}

Keywords: Napoleonaea imperialis, rind, saponin, oleanane, glycoside, napoleonaside G, napoleonaside $\mathrm{R}$

\section{INTRODUCTION}

Plants for some decades have become source of developing modern medicines for human health ${ }^{1}$. Plant usage for medicinal purpose depends on phytochemicals for its biological activities ${ }^{2,3}$. Flavonoids, alkaloids, saponins, coumarins, terpenes, tannins and glycosides are phytochemicals mostly present in plants ${ }^{4}$.

Rind is the outer covering of a fruit regarded as waste material, which is often discarded after consumption ${ }^{5}$. They are reported to have phytochemicals of pharmaceutical interest and important sources of flavour and aroma, utilized as dietary fibre, animal feed and biofuel ${ }^{6-10}$. Environmental pollution can also be reduced by converting waste materials into useful products ${ }^{11}$.

Nigerian Journal of Chemical Research
Napoleonaea imperialis, commonly called Napoleon's Hat, belongs to the family Lecythidacaea (The Brazil Nut Family), is a non-timber medicinal plant found in SouthEastern Nigeria and grows abundantly in bush fallows and secondary bushes ${ }^{12}$. The bark and fruit rind are used to treat respiratory tract infections while the twig has antimicrobial potential, hence they are used as traditional chew stick for oral hygiene ${ }^{13,14}$. The rind and seed of $N$. imperialis have antibacterial potency when evaluated against Grampositive and Gram-negative bacteria ${ }^{13}$. Neral and geranial are reported to be the major volatile compounds of the essential oil of $N$. imperialis rind, while triterpenoidal saponins have been isolated from the seed of $N$. imperialis $^{15-17}$. 


\section{MATERIALS AND METHODS}

\section{General}

All solvents used in this work were redistilled. Analytical thin layer chromatography (TLC) was conducted on silica gel (Merck $\mathrm{F}_{253}$ ) precoated aluminium plates. Vacuum liquid chromatography and column chromatography were conducted using silica gel 70-230 and 60-200 mesh ASTM (60H Merck) respectively. Detection of spots on developed TLC plates was done under UV light at wavelength of $254 \mathrm{~nm}$ and using spray reagent $\left(10 \% \mathrm{H}_{2} \mathrm{SO}_{4}-\mathrm{MeOH}\right)$ followed by heating at $105{ }^{\circ} \mathrm{C}$ for $5 \mathrm{~min}$. One dimension TLC was carried out using $5 \times 5 \mathrm{~cm}$ TLC plates and developed with various solvent systems.

All NMR data were acquired at $25^{\circ} \mathrm{C}$ on a Bruker $600 \mathrm{MHz}$ spectrometer $\left({ }^{1} \mathrm{H}, 600.06\right.$ $\mathrm{MHz}$ ) equipped with a $5 \mathrm{~mm}$ cryoprobe. The sample $(\sim 6 \mathrm{mg})$ was solubilized in $550 \mu \mathrm{L}$ methanol $\left(\mathrm{CD}_{3} \mathrm{OD}\right)$. The solution was then transferred to a $5 \mathrm{~mm}$ NMR tube. ${ }^{1} \mathrm{H}\left\{{ }^{13} \mathrm{C}\right\}$ NMR experiment was acquired with spectral width of $9090.9 \mathrm{~Hz}, 32768$ data points, 8 scans and $1.5 \mathrm{~s}$ relaxation delay. ${ }^{13} \mathrm{C} \mathrm{NMR}$ experiments was acquired with spectral width of $35714.3 \mathrm{~Hz}, 32768$ data points, 3840 scans and $1.5 \mathrm{~s}$ relaxation delay. 2D NMR data were acquired with ${ }^{1} \mathrm{H}$ spectral widths of $4424.8 \mathrm{~Hz}$ (COSY, TOCSY and NOESY), ${ }^{1} \mathrm{H} /{ }^{13} \mathrm{C}$ spectral widths of $4424.8 \mathrm{~Hz} / 22123.9 \mathrm{~Hz}$ (HSQC and HSQC-TOCSY) and ${ }^{1} \mathrm{H} /{ }^{13} \mathrm{C}$ spectral widths of $4424.8 \mathrm{~Hz} / 22123.9 \mathrm{~Hz}$ (HMBC). COSY data was acquired with 2 scans and $1536 * 768(\mathrm{t} 1 * \mathrm{t} 2)$ points, TOCSY data was acquired with 8 scans and $1024 *$ $384(\mathrm{t} 1 * \mathrm{t} 2)$ points, NOESY data was acquired with 20 scans and $1280 * 512(\mathrm{t} 1 *$ t2) points, HSQC data was acquired with 8 scans and $1024 * 768(\mathrm{t} 1 * \mathrm{t} 2)$ points, HSQCTOCSY data was acquired with 16 scans and $871 * 768(\mathrm{t} 1 * \mathrm{t} 2)$ points and HMBC data was acquired with 44 scans and $1536 * 256(\mathrm{t} 1 *$

Nigerian Journal of Chemical Research t2) points. Chemical shifts were referenced to the methanol signals at $3.35,4.78 \mathrm{ppm}\left({ }^{1} \mathrm{H}\right)$ and $49.3 \mathrm{ppm}\left({ }^{13} \mathrm{C}\right)$. All spectra were processed and analysed with MestreNova (version 14.0.1-23559).

\section{Extraction and partitioning}

Fresh fruits of $N$. imperialis were harvested from Owerri, Imo state, Nigeria. The rinds were separated from the seed, chopped, air-dried and pulverized at room temperature $\left(25^{\circ} \mathrm{C}\right)$ and weighed. Extraction of pulverized rind (617.2 g) was done using maceration with methanol for 48 hours. The extract was concentrated to dryness using a rotary evaporator (Labrota 4002 ) at $40{ }^{\circ} \mathrm{C}$ to afford a brown-sticky crude extract (148.12 g). The resulting crude methanol extract was dissolved in methanol $(200 \mathrm{ml})$ and subsequently partitioned successively between $400 \mathrm{ml} n$-hexane and $400 \mathrm{ml}$ of dichloromethane in a separatory funnel. Each of the partitioned fraction was concentrated to yield $n$-hexane fraction (11.01 g, 7.4\%), dichloromethane fraction (35.16 g, 23.7\%) and methanol fraction (101.75 g, $68.7 \%)$. These fractions were stored in the refrigerator until required for further analysis.

\section{Vacuum Liquid Chromatography}

Dichloromethane fraction (32.97 g) was weighed, and $20 \mathrm{~g}$ of silica gel added to it. The silica gel was properly mixed with the fraction and a light brown powder was obtained. The mixture was poured into a sintered glass funnel $(9.8 \mathrm{~cm} \times 10.4 \mathrm{~cm})$ already packed with $130 \mathrm{~g}$ of silica gel and was evenly distributed to the height of $4.5 \mathrm{~cm}$. Gradient elution using different combinations of $n$-hexane ( $n$-Hex), ethyl acetate (EtOAc) and methanol $(\mathrm{MeOH})$ was conducted, and fractions (200 $\mathrm{ml}$ each) collected. A total of 25 fractions were obtained and combined based on TLC analysis to afford 13 major fractions $\left(F_{1}-F_{13}\right)$. 
The dry fractions were weighed and stored in glass vials.

\section{Purification through Column Chromatography}

$F_{10}$ and $F_{11}$ which eluted with EtOAc$\mathrm{MeOH}$ (9:1) from VLC yielded a light brown solid (3.26 g) and brown powder (3.68 g) respectively after evaporation of solvents; and were subjected to purification using column chromatography. All fractions were monitored with thin layer chromatography. A packed column containing $2.3 \mathrm{~g}$ of $\mathrm{F}_{10}$ was eluted with EtOAc- $n$-Hex (6:4), EtOAc- $n$ Hex (9.5:0.5), 100\% EtOAc and EtOAc$\mathrm{MeOH}$ (9.5:0.5); and $20 \mathrm{ml}$ per fraction collected to give a total of 99 fractions. Fractions 53-71 which eluted with EtOAc$\mathrm{MeOH}$ (9.5:0.5) were combined and solvent evaporated to give isolate $\mathrm{A}(80 \mathrm{mg})$.

$\mathrm{F}_{11}(2.5 \mathrm{~g})$ was loaded in a column and eluted with EtOAc- $n$-Hex (8:2), EtOAc- $n$ Hex (9.5:0.5), EtOAc-MeOH (9.5:0.5) and EtOAc-MeOH (9.2:0.8); and $20 \mathrm{ml}$ per fraction collected to give a total of 78 fractions.

Fractions 60-76 which eluted with EtOAc$\mathrm{MeOH}$ (9.2:0.8) were combined and solvent evaporated to give golden crystals $(84 \mathrm{mg}$ ) which was subjected to further purification. For the purification of the golden crystals, a column of $30 \mathrm{~cm}$ length and $2.4 \mathrm{~cm}$ diameter was packed using EtOAc- $n$-hex (1:1) as solvent system. The column was eluted with $100 \%$ EtOAc and EtOAc-MeOH (9.5:0.5); and $5 \mathrm{ml}$ per fraction collected to give a total of 41 fractions. Fractions 16-39 eluted with EtOAc-MeOH (9.05:0.5) were combined and solvent evaporated to give isolate $B(45 \mathrm{mg})$.

Isolates A and B (5 mg each) were separately dissolved in dichloromethane (I $\mathrm{ml}$ ) and tested with freshly prepared Liebermann-Burchard reagent (1 ml). Purple colouration was observed for both reactions.

\section{RESULTS AND DISCUSSION}

Isolates $\mathrm{A}$ and $\mathrm{B}$ showed positive reaction to Liebermann-Burchard test with purple colouration (Table 1) indicating the presence of triterpenoidal saponins 17,18 . Visibility of the two compounds under UV light of wavelength $252 \mathrm{~nm}$ (Table 1) revealed the existence of a conjugated diene while the purple spots seen after developed plates were sprayed with $10 \%$ sulphuric acid in methanol reagent and heated indicated the presence of terpenoids ${ }^{19-21}$.

Table 1. Profile of isolates A and B

\begin{tabular}{lllllll}
\hline Isolate & $\begin{array}{l}\text { Spot } \\
\text { under } \\
\text { UV }\end{array}$ & $\begin{array}{l}\text { Spot after } \\
\text { MeOH-H } \\
\text { reagent +heat }\end{array}$ & TLC mobile phase & $\begin{array}{l}\mathbf{R}_{\mathbf{f}} \\
\text { value }\end{array}$ & $\begin{array}{l}\text { Liebermann- } \\
\text { Burchard } \\
\text { test }\end{array}$ & $\begin{array}{l}\text { Percentage } \\
\text { yield }\end{array}$ \\
\hline A (Crystal) & Purple & Purple & $9: 1$ EtOAc- MeOH & 0.6 & Purple & 0.2 \\
B (Crystal) & Purple & Purple & $9: 1$ Acetone- MeOH & 0.9 & Purple & 0.1 \\
\hline
\end{tabular}

Structures of isolates A (1) and B (2) were determined using ${ }^{1} \mathrm{H}$ and ${ }^{13} \mathrm{C}$ NMR experiments aided by connectivity observed in two-dimension NMR experiments (Tables 2 and 3 ).

Nigerian Journal of Chemical Research
${ }^{13} \mathrm{C}$ NMR spectrum of isolate A showed fifty-two carbon signals. Two carbon signals at $\delta 170.6\left(\mathrm{C}-1^{*}\right)$ and $\delta 168.5\left(\mathrm{C}-1^{* * *}\right)$ were identified as carbonyl (esters) carbons. Ten ring residue signals, $\delta 74.7\left(\mathrm{C}-2^{\prime}\right), \delta 73.9(\mathrm{C}$ 3'), $\delta 71.0$ (C-4'), $\delta 71.6$ (C-5'), $\delta 61.70\left(\mathrm{C}^{\prime} 6^{\prime}\right)$, 
$\delta 76.6$ (C-2"), 871.8 (C-3"), 891.2 (C-4"), $\delta 72.7$ (C-5"), $\delta 66.4$ (C-6") belonging to the sugar moiety as well as two anomeric carbons, $\delta 105.0$ (C-1') and $\delta 104.2(\mathrm{C}-1 ")$, were also identified. ${ }^{1} \mathrm{H}$ NMR spectrum of isolate $\mathrm{A}$ showed two anomeric protons at $\delta 4.57\left(\mathrm{H}-1^{\prime}\right)$ and $\delta 4.77(\mathrm{H}-1 ")$. The assignments of isolate $\mathrm{A}{ }^{1} \mathrm{H}$ and ${ }^{13} \mathrm{C}$ signals using HSQC and verified with ${ }^{1} \mathrm{H}-{ }^{1} \mathrm{H}$ COSY (Table 2) show eight quaternary carbon signals, $\delta 47.5(\mathrm{C}-4)$, $\delta 44.5$ (C-8), $\delta 35.7$ (C-10), $\delta 139.7$ (C-13), $\delta 42.5(\mathrm{C}-14), \delta 42.2(\mathrm{C}-17), \delta 139.1$ (C-18) and $\delta 37.5$ (C-20); six primary carbon (methyl groups) signals, $\delta 16.2(\mathrm{C}-23), \delta 16.2(\mathrm{C}-25)$, $\delta 16.5$ (C-26), $\delta 14.1$ (C-27), $\delta 18.5$ (C-29) and $\delta 19.9$ (C-30); ten secondary carbon (methylenes) signals, 839.2 (C-1), $\delta 26.9$ (C2), $\delta 27.1$ (C-6), $\delta 31.6$ (C-7), $\delta 33.0$ (C-15), $\delta 34.5$ (C-16), $\delta 24.9$ (C-19), $\delta 34.0$ (C-22), $\delta 64.2(\mathrm{C}-24)$ and $\delta 66.2(\mathrm{C}-28)$; six tertiary carbon signals at $\delta 80.5(\mathrm{C}-3), \delta 40.3(\mathrm{C}-5)$, $\delta 41.0(\mathrm{C}-9), \delta 125.8(\mathrm{C}-11), \delta 124.2(\mathrm{C}-12)$ and $\delta 68.8(\mathrm{C}-21)$ were assigned to the aglycone. An ester carbonyl carbon, 170.6 (C-1*), quaternary carbon, $\delta 142.1\left(\mathrm{C}-2^{*}\right)$, primary carbons, $\delta 22.10\left(\mathrm{C}-3^{*}\right)$ and $\delta 15.8\left(\mathrm{C}-5^{*}\right)$, and tertiary carbon $\delta 130.8\left(\mathrm{C}-4^{*}\right)$ indicated the presence of an angeloyl group (angeloyl 1), while the ester carbonyl carbon signal at $\delta 168.5\left(\mathrm{C}-1^{* *}\right)$, quaternary carbon $\delta 128.7$ (C$\left.2^{* *}\right)$, primary carbons $\delta 20.09\left(\mathrm{C}-3^{* *}\right), 15.8(\mathrm{C}$ $\left.5^{* *}\right)$ and tertiary carbon $\delta 140.0\left(\mathrm{C}-4^{* *}\right)$ showed the presence of another angeloyl group (angeloyl 2). From HSQC spectrum, eight proton signals observed at $\delta 4.52\left(\mathrm{H}-^{\mathrm{a}} \mathrm{O}\right)$, $\delta 4.54\left(\mathrm{H}^{\mathrm{b}}{ }^{\mathrm{O}} \mathrm{O}\right), \delta 3.81\left(\mathrm{H}^{\mathrm{c}} \mathrm{O}\right), \delta 4.75\left(\mathrm{H}^{\mathrm{d}}{ }^{\mathrm{d}} \mathrm{O}\right)$, $\delta 4.48\left(\mathrm{H}-^{-} \mathrm{O}\right), \delta 3.76\left(\mathrm{H}-^{\mathrm{f}} \mathrm{O}\right), \delta 3.61\left(\mathrm{H}^{\mathrm{g}}{ }^{\mathrm{g}} \mathrm{O}\right)$ and $\delta 3.78\left(\mathrm{H}^{-}{ }^{\mathrm{h}} \mathrm{O}\right)$ were not attached to any carbon in the HSQC spectrum and were assigned as the sugar hydroxy protons.

Olefinic carbon signals at $\delta 125.8(\mathrm{C}-11)$, $\delta 124.2$ (C-12) supported by proton signals at $\delta 5.40(\mathrm{H}-11)$ and $\delta 5.32(\mathrm{H}-12)$ and two quaternary carbons at $\delta 139.1(\mathrm{C}-13)$ and $\delta 139.7$ (C-18) indicate the existence of a heteroannular diene in the structure of isolate A. These signals are similar to the diene peaks of saikosaponin [22]. The additional quaternary carbons at $\delta 47.5(\mathrm{C}-4), \delta 44.5(\mathrm{C}$ 8), $\delta 35.7$ (C-10), $\delta 42.5$ (C-14), $\delta 42.2$ (C-17) and $\delta 37.5(\mathrm{C}-20)$ have been reported for saikosaponin isolated from Bupleurum chinense, and derivatives of saikosaponin 22, ${ }^{23}$. Signals of the angeloyl groups $\delta 170.6(\mathrm{C}$ $\left.1^{*}\right), \delta 168.5\left(\mathrm{C}-1^{* *}\right), \delta 142.1\left(\mathrm{C}-2^{*}\right), \delta 128.7(\mathrm{C}-$ $\left.2^{* *}\right), \delta 1.98\left(\mathrm{H}-3^{*}\right), \delta 1.82\left(\mathrm{H}-3^{* *}\right), \delta 6.12\left(\mathrm{H}-4^{*}\right)$ $\delta 6.22\left(\mathrm{H}-4^{* *}\right), \delta 2.10\left(\mathrm{H}-5^{*}\right)$ and $\delta 1.95\left(\mathrm{H}-5^{* *}\right)$ were similar to angeloyl reported by other researchers ${ }^{17,24}$. The proton signals at $\delta 1.98$ $\left(\mathrm{H}-3^{*}\right)$ and $\delta 1.82\left(\mathrm{H}-3^{* *}\right)$ of the angeloyl groups correlated to $\delta 4.21(\mathrm{H}-21)$ and $\delta 4.28$ (H-28) of the aglycone respectively (Figure 1) indicating the positions where the angeloyl groups are attached to the aglycone. Therefore, the aglycone was characterized as 21,28-diangeloyloxy-24-hydroxy-olean-

$11,13(18)$-diene. The anomeric proton $\delta 4.57$ $\left(\mathrm{H}-1^{\prime}\right)$ was found to correlate to $\delta 3.58(\mathrm{H}-3)$ which was directly correlated to $\delta 80.5$ (C-3) of the aglycone while the anomeric proton $\delta 4.77(\mathrm{H}-1 ")$ correlated with $\delta 3.21$ (H-2') of the sugar moiety. These correlations show the position of attachment of the sugar moiety to the aglycone and the connectivity of all the protons in the sugar moiety. The sugar moiety of isolate A was characterized as 3-O-[Dglucopyranosyl $(1 \rightarrow 2)]$-D-glucopyranoside.

The proposed name for isolate A (1), Napoleonaside G, is 3-O-[Dglucopyranosyl $(1 \rightarrow 2)$-D-glucopyranosyl]21,28-diangeloyloxy-24-hydroxy-olean11,13(18)-diene. 
Table 2. ${ }^{13} \mathrm{C}$ and ${ }^{1} \mathrm{H}$ chemical shift data of isolate A (Napoleonaside $\mathrm{G}$ ) in $\mathrm{CD}_{3} \mathrm{OD}$

\begin{tabular}{|c|c|c|c|c|c|}
\hline \multicolumn{3}{|r|}{ Aglycone } & \multicolumn{3}{|r|}{ Sugar moiety } \\
\hline Position & $\delta^{13} \mathrm{C}$ & $\delta^{1} \mathbf{H}$ & Position & $\delta^{13} \mathrm{C}$ & $\delta^{1} \mathbf{H}$ \\
\hline 1 & 39.2 & $1.67(1 \mathrm{H}, \mathrm{m}), 0.94(1 \mathrm{H}, \mathrm{m})$ & $1^{\prime}$ & 105.0 & $4.57(1 \mathrm{H}, \mathrm{d}, 3.0 \mathrm{~Hz})$ \\
\hline 2 & 26.9 & $1.98(1 \mathrm{H}, \mathrm{s}), 1.82(1 \mathrm{H}, \mathrm{s})$ & $2^{\prime}$ & 74.7 & $3.21(1 \mathrm{H}, \mathrm{m})$ \\
\hline 3 & 80.5 & $3.58(1 \mathrm{H}, \mathrm{m})$ & $3^{\prime}$ & 73.9 & $5.06(1 \mathrm{H}, \mathrm{m})$ \\
\hline 4 & 47.5 & - & $4^{\prime}$ & 71.0 & $3.82(1 \mathrm{H}, \mathrm{t}, 3.0 \mathrm{~Hz})$ \\
\hline 5 & 40.3 & $2.54(1 \mathrm{H}, \mathrm{t}, 3.0 \mathrm{~Hz})$ & $5^{\prime}$ & 71.6 & $5.40(1 \mathrm{H}, \mathrm{m})$ \\
\hline 6 & 27.1 & $1.45(1 \mathrm{H}, \mathrm{m}), 1.36(1 \mathrm{H}, \mathrm{d}, 6.0 \mathrm{~Hz})$ & $6^{\prime}$ & 61.7 & $3.79(1 \mathrm{H}, \mathrm{t}, 1.5 \mathrm{~Hz}), 3.72(1 \mathrm{H}, \mathrm{q}, 3.0 \mathrm{~Hz})$ \\
\hline 7 & 31.6 & $1.67(1 \mathrm{H}, \mathrm{m}), 1.45(1 \mathrm{H}, \mathrm{m})$ & $1 "$ & 104.2 & $4.77(1 \mathrm{H}, \mathrm{d}, 3.0 \mathrm{~Hz})$ \\
\hline 8 & 44.5 & - & 2" & 76.6 & $3.48(1 \mathrm{H}, \mathrm{t}, 1.5 \mathrm{~Hz})$ \\
\hline 9 & 41.0 & $2.36(1 \mathrm{H}, \mathrm{d}, 6.0 \mathrm{~Hz})$ & 3" & 71.8 & $3.88(1 \mathrm{H}, \mathrm{d}, 3.0 \mathrm{~Hz})$ \\
\hline 10 & 35.7 & - & $4 "$ & 91.2 & $4.15(1 \mathrm{H}, \mathrm{s})$ \\
\hline 11 & 125.8 & $5.40(1 \mathrm{H}, \mathrm{m})$ & $5 "$ & 72.7 & $3.58(1 \mathrm{H}, \mathrm{m})$ \\
\hline 12 & 124.2 & $5.32(1 \mathrm{H}, \mathrm{m})$ & 6" & 66.4 & $3.42(1 \mathrm{H}, \mathrm{m}), 3.21(1 \mathrm{H}, \mathrm{m})$ \\
\hline 13 & 139.7 & - & ${ }^{\mathrm{a}} \mathrm{O}$ & & $4.52(1 \mathrm{H}, \mathrm{t}, 1.5 \mathrm{~Hz})$ \\
\hline 14 & 42.5 & - & ${ }^{\mathrm{b}} \mathrm{O}$ & & $4.54(1 \mathrm{H}, \mathrm{s})$ \\
\hline 15 & 33.0 & $1.30(1 \mathrm{H}, \mathrm{d}, 3.0 \mathrm{~Hz}), 1.98(1 \mathrm{H}, \mathrm{s})$ & ${ }^{\mathrm{c}} \mathrm{O}$ & & $3.81(1 \mathrm{H}, \mathrm{s})$ \\
\hline 16 & 34.5 & $1.78(1 \mathrm{H}, \mathrm{t}, 1.5 \mathrm{~Hz}), 1.36(1 \mathrm{H}, \mathrm{d}, 6.0 \mathrm{~Hz})$ & ${ }^{\mathrm{d}} \mathrm{O}$ & & $4.75(1 \mathrm{H}, \mathrm{s})$ \\
\hline 17 & 42.2 & - & ${ }^{\mathrm{e}} \mathrm{O}$ & & $4.48(1 \mathrm{H}, \mathrm{s})$ \\
\hline 18 & 139.1 & - & ${ }^{\mathrm{f}} \mathrm{O}$ & & $3.76(1 \mathrm{H}, \mathrm{d}, 3.0 \mathrm{~Hz})$ \\
\hline 19 & 24.9 & $1.92(1 \mathrm{H}, \mathrm{s}), 1.90(1 \mathrm{H}, \mathrm{d}, 3 \mathrm{~Hz})$ & ${ }^{\mathrm{g}} \mathrm{O}$ & & $3.61(1 \mathrm{H}, \mathrm{d}, 3.0 \mathrm{~Hz})$ \\
\hline 20 & 37.5 & - & ${ }^{\mathrm{h}} \mathrm{O}$ & & $3.78(1 \mathrm{H}, \mathrm{t}, 1.5 \mathrm{~Hz})$ \\
\hline 21 & 68.8 & $4.21(1 \mathrm{H}, \mathrm{m})$ & Angeloyl 1 & & \\
\hline 22 & 34.0 & $1.67(1 \mathrm{H}, \mathrm{s}), 1.36(1 \mathrm{H}, \mathrm{d}, 6.0 \mathrm{~Hz})$ & $1^{*}-x^{*}$ & 170.6 & - \\
\hline 23 & 16.2 & $1.21(3 \mathrm{H}, \mathrm{s})$ & $2^{*}$ & 142.1 & - \\
\hline 24 & 64.2 & $4.09(1 \mathrm{H}, \mathrm{t}, 3 \mathrm{~Hz}), 3.21(1 \mathrm{H}, \mathrm{d}, 3 \mathrm{~Hz})$ & $3^{*}$ & 15.8 & $1.98(3 \mathrm{H}, \mathrm{s})$ \\
\hline 25 & 16.2 & $1.15(3 \mathrm{H}, \mathrm{s})$ & $4^{*}$ & 130.8 & $6.12(1 \mathrm{H}, \mathrm{q}, 6.0 \mathrm{~Hz})$ \\
\hline 26 & 16.5 & $1.06(3 \mathrm{H}, \mathrm{s})$ & $5^{*}$ & 22.1 & $2.10(3 \mathrm{H}, \mathrm{s})$ \\
\hline 27 & 14.1 & $0.94(3 \mathrm{H}, \mathrm{s})$ & Angeloyl 2 & & \\
\hline 28 & 66.2 & $4.28(1 \mathrm{H}, \mathrm{s}), 3.37(1 \mathrm{H}, \mathrm{m})$ & $1^{* *}$ & 168.5 & - \\
\hline 29 & 18.5 & $1.03(3 \mathrm{H}, \mathrm{s})$ & $2^{* *}$ & 128.7 & - \\
\hline \multirow[t]{3}{*}{30} & 19.9 & $0.98(3 \mathrm{H}, \mathrm{s})$ & $3^{* *}$ & 20.1 & $1.82(3 \mathrm{H}, \mathrm{s})$ \\
\hline & & & $4^{* *}$ & 140.0 & $6.22(1 \mathrm{H}, \mathrm{q}, 6.0 \mathrm{~Hz})$ \\
\hline & & & $5^{* *}$ & 15.8 & $1.95(3 \mathrm{H}, \mathrm{s})$ \\
\hline
\end{tabular}




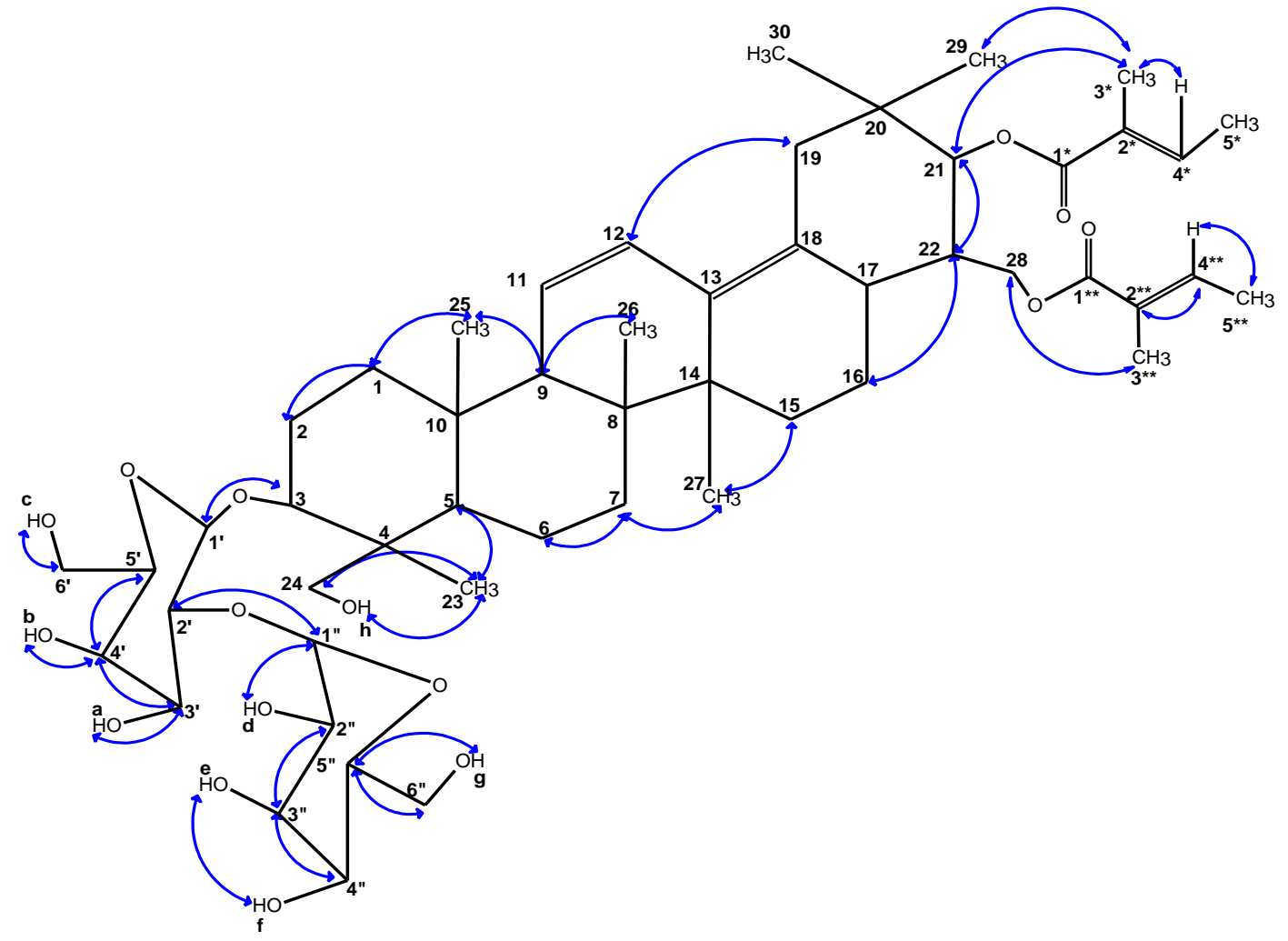

Figure 1. Structure and numbering of isolate A (Napoleonaside G) used in this report. COSY correlations are indicated with blue arrows.

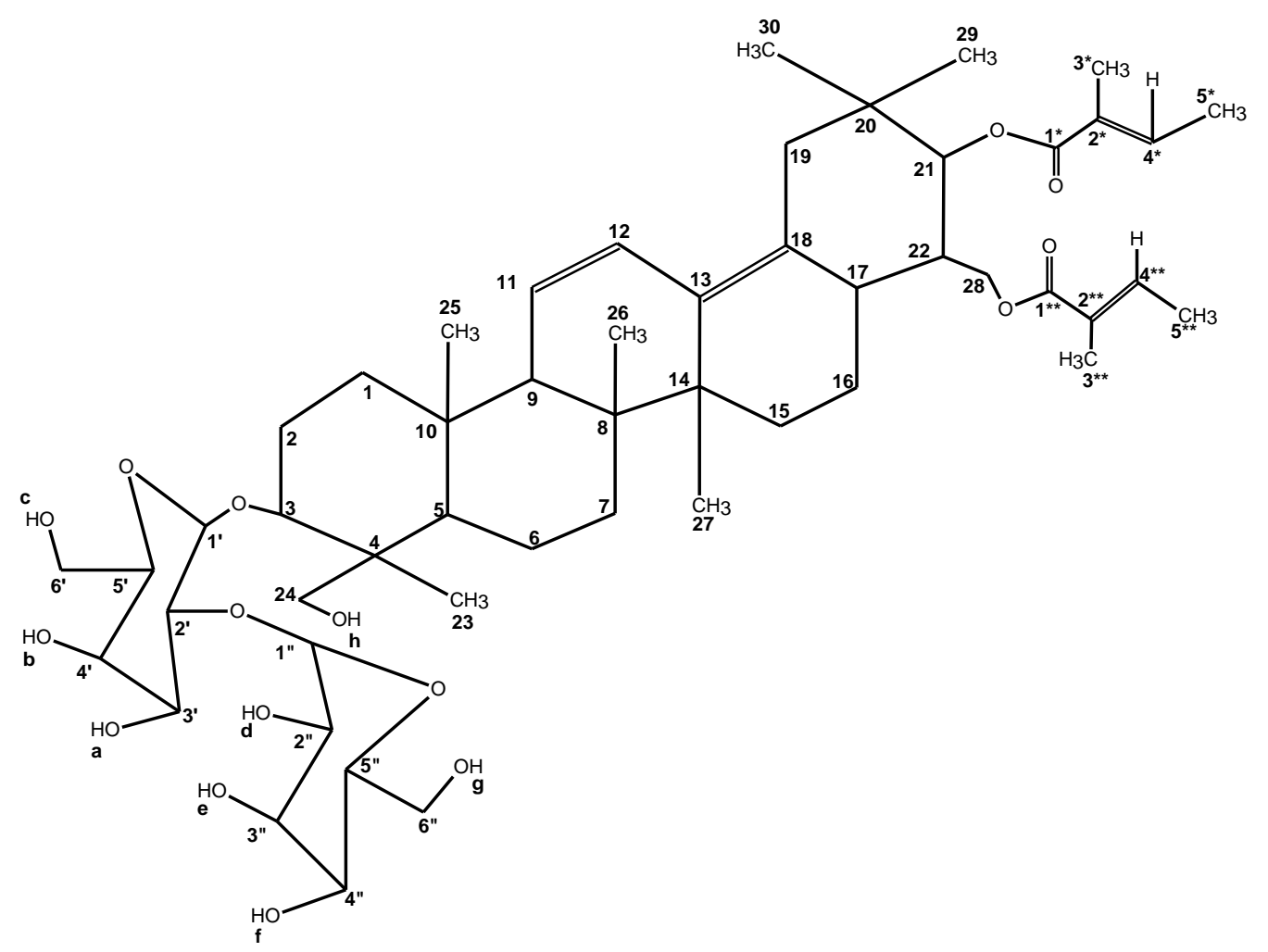


Assignment of ${ }^{1} \mathrm{H}$ and ${ }^{13} \mathrm{C}$ peaks of isolate $\mathrm{B}$ aided by $2 \mathrm{D}$ NMR experiments (Table 3 ) had similarities to isolate A (Table 2). Long range connectivity from HMBC showed a correlation between $\delta 170.7\left(\mathrm{C}-1^{*}\right)$ of the angeloyl group and $\delta 68.8(\mathrm{C}-21)$ which is directly correlated to $\delta 4.20$ (H-21) (Figure 2). The aglycone of isolate B was characterized as 21-angeloyloxy-24,28dihydroxy-olean-11,13(18)-diene.

The correlation between anomeric proton $\mathrm{H}-1^{\prime}$ and $\mathrm{C}-3$ of the aglycone, $\mathrm{C}-1$ " and $\mathrm{H}-2$ " of the sugar moiety were established from $2 \mathrm{D}$ connectivity. The signal at $\delta 91.9$ (C-3) which is directly correlated with a $\delta 3.19(\mathrm{H}-3)$ in HSQC (Table 3 ) show a long-range correlation with the proton signal $\delta 4.32\left(\mathrm{H}-1^{\prime}\right)$ which is directly correlated with the anomeric carbon $\delta 104.8\left(\mathrm{C}-1^{\prime}\right) .{ }^{13} \mathrm{C}$ signal at $\delta 105.8$ (C-1") exhibited a long-range correlation with ${ }^{1} \mathrm{H}$ peak at $\delta 5.03$ (H-2"). HMBC of isolate $\mathrm{B}$ (Figure 2) shows a strong correlation between $\delta 168.8\left(\mathrm{C}-1^{* *}\right)$ of an angeloyl group and $\delta 5.03\left(\mathrm{H}-2^{\prime \prime}\right)$ which is directly correlated with $\delta 74.4$ (C-2") of the sugar moiety. This type of correlation of an angeloyl group to a sugar moiety has been reported in pentacyclic triterpenoid saponin isolated from the husks of Xanthoceras sorbifolium Bunge and sesquiterpenes from the fruits of Pittosporum undulatum ${ }^{25,26}$. The sugar moiety of isolate $\mathrm{B}$ was characterized as 3-O-[Dglucopyranosyl $(1 \rightarrow 4)$-D-glucopyranoside-2"angeloyl. The proposed name for isolate B (2), Napoleonaside $\mathrm{R}, \quad$ is 3-O-[Dglucopyranosyl $(1 \rightarrow 4)$-D-glucopyranosyl-2"angeloyl]-21-angeloyloxy-24,28-dihydroxyolean-11, 13(18)-diene.

Glycosides with oleanane-type triterpenoid sapogenin are reported to show antioxidant and anti-diabetic potentials, antimicrobial, antiinflammatory, cytotoxic activities and are used as tools in cancer therapy ${ }^{27-31}$.

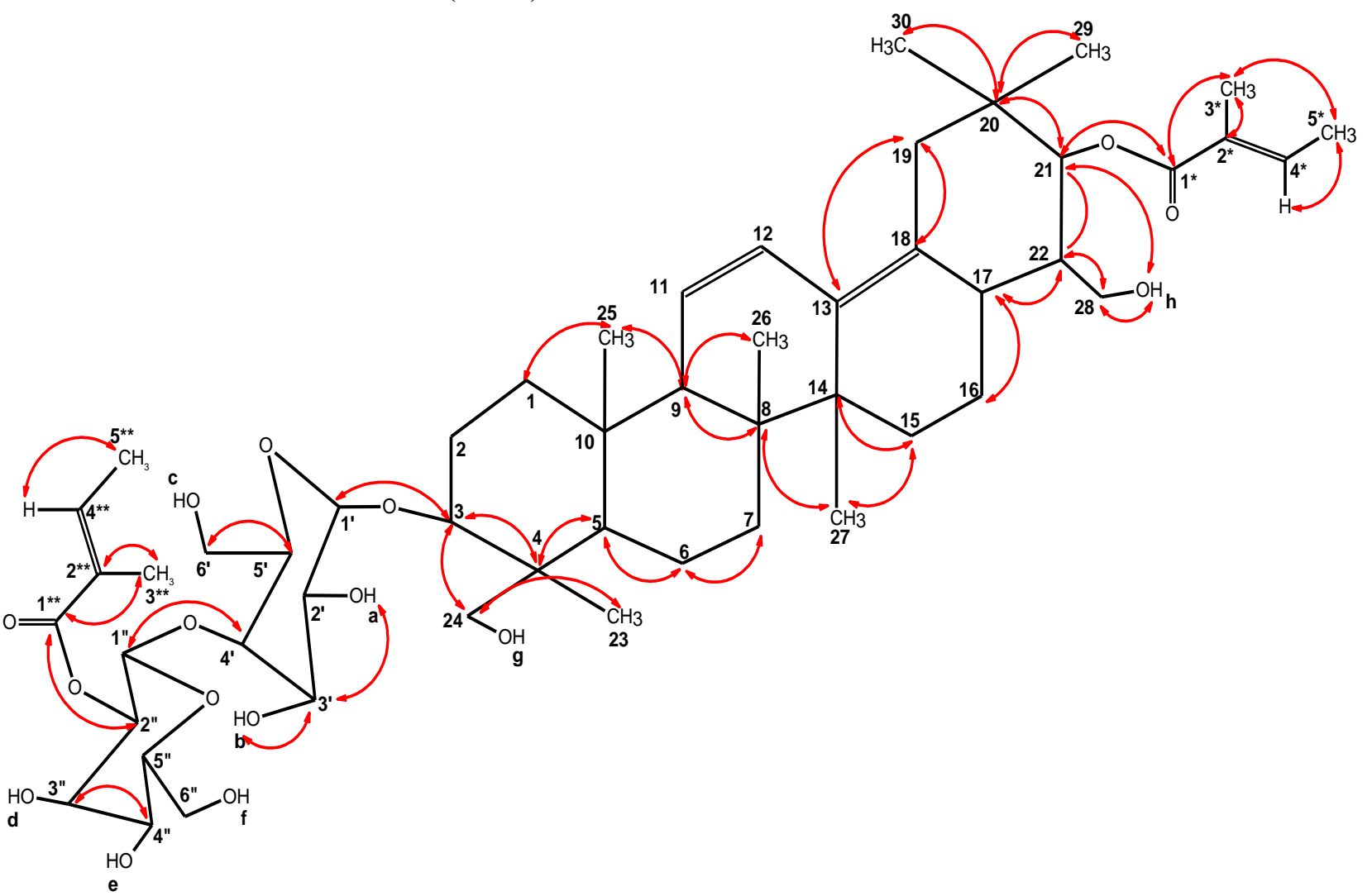


Figure 2. Structure and numbering of isolate B (Napoleonaside R) used in this report. HMBC correlations are indicated with red arrows

Table 3. ${ }^{13} \mathrm{C}$ and ${ }^{1} \mathrm{H}$ chemical shift data of isolate B (Napoleonaside R) in $\mathrm{CD}_{3} \mathrm{OD}$

\begin{tabular}{|c|c|c|c|c|c|}
\hline \multicolumn{3}{|r|}{ Aglycone } & \multicolumn{3}{|c|}{ Sugar moiety } \\
\hline Position & $\delta^{13} \mathrm{C}$ & $\delta^{1} \mathbf{H}$ & Position & $\delta^{13} \mathrm{C}$ & $\delta^{1} \mathbf{H}$ \\
\hline 1 & 39.5 & $1.58(1 \mathrm{H}, \mathrm{s}), 1.03(1 \mathrm{H}, \mathrm{s})$ & $1^{\prime}$ & 104.8 & $4.32(1 \mathrm{H}, \mathrm{m})$ \\
\hline 2 & 26.9 & $1.82(1 \mathrm{H}, \mathrm{s}), 1.67(1 \mathrm{H}, \mathrm{d}, 3.0 \mathrm{~Hz})$ & $2^{\prime}$ & 72.9 & $3.82(1 \mathrm{H}, \mathrm{m})$ \\
\hline 3 & 91.9 & $3.19(1 \mathrm{H}, \mathrm{t}, 3 \mathrm{~Hz})$ & $3^{\prime}$ & 75.7 & $5.23(1 \mathrm{H}, \mathrm{m})$ \\
\hline 4 & 40.9 & - & $4^{\prime}$ & 77.5 & $3.58(1 \mathrm{H}, \mathrm{m})$ \\
\hline 5 & 48.1 & $2.56(1 \mathrm{H}, \mathrm{t}, 3.0 \mathrm{~Hz})$ & $5^{\prime}$ & 74.0 & $3.48(1 \mathrm{H}, \mathrm{m})$ \\
\hline 6 & 27.4 & $1.48(1 \mathrm{H}, \mathrm{s}) 1.43(1 \mathrm{H}, \mathrm{m})$ & $6^{\prime}$ & 67.1 & $3.23(1 \mathrm{H}, \mathrm{m}), 3.37(1 \mathrm{H}, \mathrm{m})$ \\
\hline 7 & 45.5 & $1.67(1 \mathrm{H}, \mathrm{d}, 3.0 \mathrm{~Hz}), 1.58(1 \mathrm{H}, \mathrm{s})$ & $1 "$ & 105.8 & $4.57(\mathrm{~m})$ \\
\hline 8 & 37.6 & - & $2 "$ & 74.4 & $5.03(1 \mathrm{H}, \mathrm{m})$ \\
\hline 9 & 40.9 & $2.38(\mathrm{~m})$ & $3 "$ & 80.4 & $3.78(1 \mathrm{H}, \mathrm{m})$ \\
\hline 10 & 47.5 & - & $4 "$ & 71.3 & $5.40(1 \mathrm{H}, \mathrm{q}, 3.0 \mathrm{~Hz})$ \\
\hline 11 & 124.5 & $5.32(1 \mathrm{H}, \mathrm{t}, 1.5 \mathrm{~Hz})$ & $5 "$ & 74.4 & $4.01(1 \mathrm{H}, \mathrm{m})$ \\
\hline 12 & 125.9 & $5.40(1 \mathrm{H}, \mathrm{q}, 3.0 \mathrm{~Hz})$ & $6 "$ & 64.4 & $3.65(1 \mathrm{H}, \mathrm{m}), 3.48(1 \mathrm{H}, \mathrm{m})$ \\
\hline 13 & 139.7 & - & ${ }^{\mathrm{a}} \mathrm{O}$ & & $3.66(1 \mathrm{H}, \mathrm{d}, 3.0 \mathrm{~Hz})$ \\
\hline 14 & 42.6 & - & ${ }^{\mathrm{b}} \mathrm{O}$ & & $3.72(1 \mathrm{H}, \mathrm{s})$ \\
\hline 15 & 34.6 & $1.82(1 \mathrm{H}, \mathrm{s}), 1.38(1 \mathrm{H}, \mathrm{s})$ & ${ }^{\mathrm{c}} \mathrm{O}$ & & $3.89(1 \mathrm{H}, \mathrm{s})$ \\
\hline 16 & 20.2 & $1.67(1 \mathrm{H}, \mathrm{d}, 3.0 \mathrm{~Hz}), 1.43(1 \mathrm{H}, \mathrm{m})$ & ${ }^{\mathrm{d}} \mathrm{O}$ & & $4.89(1 \mathrm{H}, \mathrm{d}, 3.0 \mathrm{~Hz})$ \\
\hline 17 & 42.2 & - & ${ }^{\mathrm{e}} \mathrm{O}$ & & $4.48(1 \mathrm{H}, \mathrm{t}, 1.5 \mathrm{~Hz})$ \\
\hline 18 & 139.2 & - & ${ }^{\mathrm{f}} \mathrm{O}$ & & $4.72(1 \mathrm{H}, \mathrm{m})$ \\
\hline 19 & 24.8 & $1.90(1 \mathrm{H}, \mathrm{d}, 3.0 \mathrm{~Hz}), 1.92(1 \mathrm{H}, \mathrm{s})$ & $\mathrm{g}_{\mathrm{O}}$ & & $3.63(1 \mathrm{H}, \mathrm{s})$ \\
\hline 20 & 37.6 & - & ${ }^{\mathrm{h}} \mathrm{O}$ & & $3.88(1 \mathrm{H}, \mathrm{s})$ \\
\hline 21 & 68.8 & $4.20(1 \mathrm{H}, \mathrm{q}, 3 \mathrm{~Hz})$ & Angeloyl 1 & & \\
\hline 22 & 31.6 & $1.67(1 \mathrm{H}, \mathrm{d}, 3.0 \mathrm{~Hz}), 1.43(1 \mathrm{H}, \mathrm{m})$ & $1^{*}$ & 170.7 & - \\
\hline 23 & 16.2 & $1.19(3 \mathrm{H}, \mathrm{s})$ & $2^{*}$ & 141.9 & - \\
\hline 24 & 70.3 & $4.07(1 \mathrm{H}, \mathrm{m}), 3.78(1 \mathrm{H}, \mathrm{s})$ & $3^{*}$ & 22.6 & $2.10(3 \mathrm{H}, \mathrm{s})$ \\
\hline 25 & 18.6 & $1.03(3 \mathrm{H}, \mathrm{s})$ & $4^{*}$ & 140.0 & $6.22(1 \mathrm{H}, \mathrm{q}, 6.0 \mathrm{~Hz})$ \\
\hline 26 & 19.4 & $1.05(3 \mathrm{H}, \mathrm{s})$ & $5^{*}$ & 15.9 & $1.98(3 \mathrm{H}, \mathrm{s})$ \\
\hline 27 & 14.1 & $0.94(3 \mathrm{H}, \mathrm{s})$ & Angeloyl 2 & & \\
\hline 28 & 66.2 & $4.25(1 \mathrm{H}, \mathrm{m}), 4.15(1 \mathrm{H}, \mathrm{m})$ & $1^{* *}$ & 168.8 & - \\
\hline
\end{tabular}




\begin{tabular}{llllll}
29 & 16.9 & $0.94(3 \mathrm{H}, \mathrm{s})$ & $2^{* *}$ & 128.5 & - \\
30 & 16.9 & $1.03(3 \mathrm{H}, \mathrm{s})$ & $3^{* *}$ & 20.5 & $1.82(3 \mathrm{H}, \mathrm{s})$ \\
& & $4^{* *}$ & 140.3 & $6.12(1 \mathrm{H}, \mathrm{q}, 6.0 \mathrm{~Hz})$ \\
& & $5^{* *}$ & 15.8 & $1.95(3 \mathrm{H}, \mathrm{s})$ \\
\hline
\end{tabular}

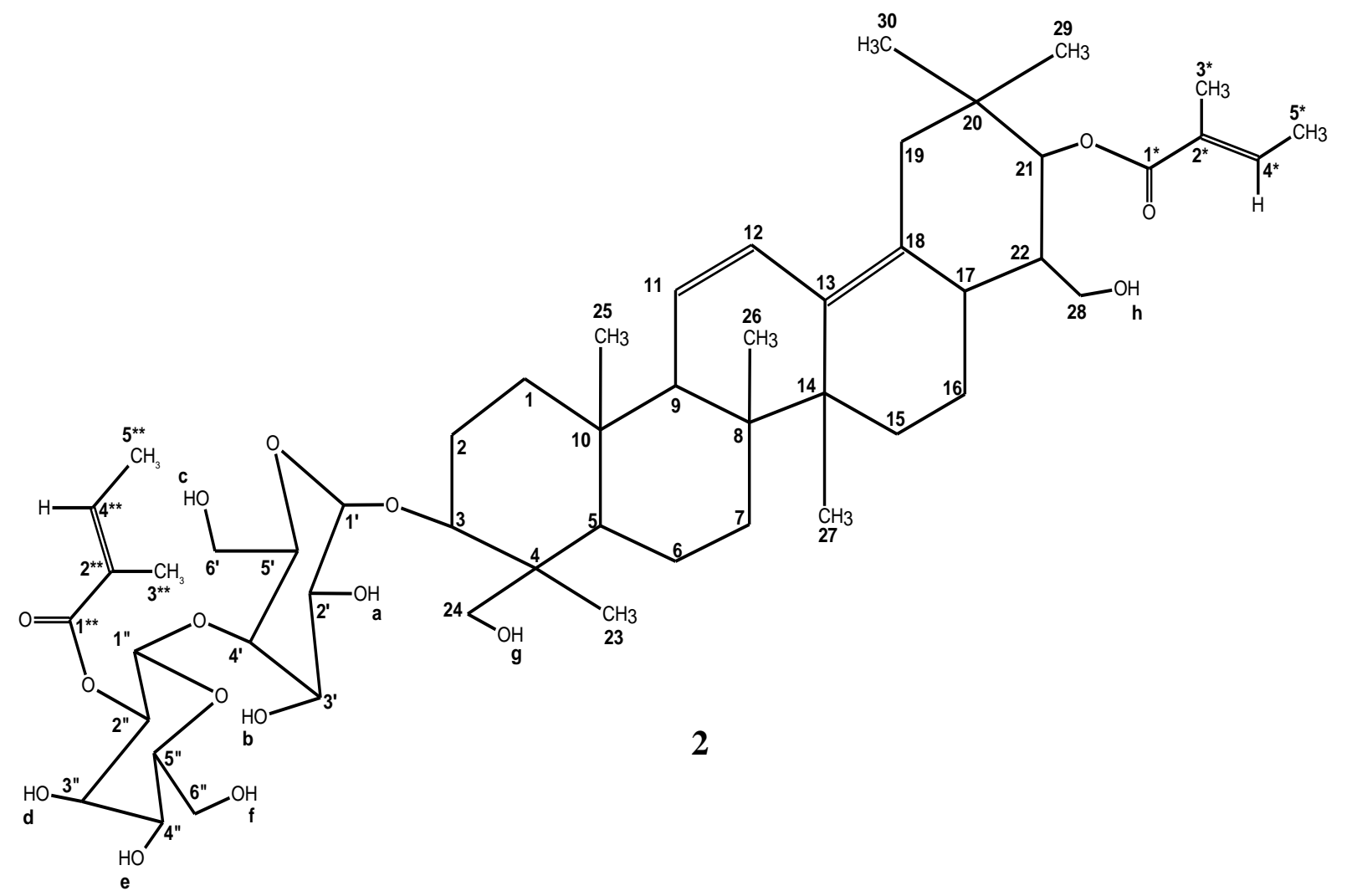

\section{CONCLUSION}

Two new oleanane-type glycosides, Napoleonaside G and Napoleonaside R, proposed as 3-O-[D-glucopyranosyl $(1 \rightarrow 2)$-Dglucopyranosyl]-21,28-diangeloyloxy-24hydroxy-olean-11,13(18)-diene and 3-O-[Dglucopyranosyl $(1 \rightarrow 4)$-D-glucopyranosyl-2"angeloyl]-21-angeloyloxy-24,28-dihydroxy- olean-11,13(18)-diene respectively were successfully isolated from the rind of Napoleonaea imperialis.

\section{ACKNOWLEDGMENTS}

The authors are grateful to the Tertiary Education Trust Fund (TETFund), Nigeria for funding this research. 


\section{REFERENCES}

1. Prasad, D. M., Izam, A, Khan, R., Jatropha curcas: Plant of medicinal benefits, Journal of Medicinal Plants Research, 2012, 6(14), 2691-2699.

2. Belkacem, N., Djaziri, R., Lahfa, F., ElHaci, I. A., Boucherit, Z., Phytochemical screening and in vitro antioxidant activity of various Punica granatum Linn. peel extracts from Algeria: A comparative study, Phytotherapie, 2013, 12(6), 372-379.

3. Elango, R., Jadhav, U., Phytochemical screening of Moringa oleifera using high performance thin layer chromatography, Plant Archives, 2010, 10(2), 49-51.

4. Sharma, V., Paliwal, R., Preliminary phytochemical investigation and thin layer chromatography profiling of sequential extracts of Moringa oleifera pods, International Journal of Green Pharmacy, 2013, 7, 41-45.

5. Asyifah, M. R., Lu, K., Ting, H., Zhang, D., Hidden potential of tropical fruit waste components as a useful source of remedy for obesity, Journal of Agriculture and Food chemistry, 2014, 62 ,

3505-3516.

6. EI-Hawary, S. S., Rabeh, A. M., Mangifera indica peels: A common waste product with impressive immunostimulant, anticancer and antimicrobial potency, Journal of Natural Sciences Research, 2014, 4(2), 102-115.
7. Saleem, M., Saeed, M., Potential application of waste fruit peels (orange, yellow lemon and banana) as wide range natural antimicrobial agent, Journal of King Saud University-Science, 2020, 32(1),

805-810.

8. Naganathan, K., Thirunavukkarasu, S., Green way genesis of silver nanoparticles using multiple fruit peels waste and its antimicrobial, antioxidant and anti-tumor cell line studies, Materials Science and Engineering, 2017, 10, 191-198.

9. Sudha, M. L., Baskaran, V., Leelavathi, $\mathrm{K}$., Apple pomace as a source of dietary fiber and polyphenols and its effect on the rheological characteristics and cake making, Food Chemistry, 2007, 104, 686-690.

10. Sharma, K., Mahato, N., Cho, H. M., Lee, Y. R., Converting citrus waste into value added products: Economic and environment friendly approaches, Nutrition, 2017, 34, 29-46.

11. Arora, M., Kaur, P., Phytochemical screening of orange peel and pulp, International Journal of Research in Engineering and Technology, 2013, 2(12), $517-521$.

12. James, O., Friday, E. A., Unekwuojo, E. G., Antihypertensive effect of methanol extract of Napoleonaea imperialis (P. Beauv) in adrenaline induced hypertensive albino Rats, International Journal of Biochemistry Research \& Review, 2011, 1(2), 47-57.

13. Ndukwe, G. I., Ojinnaka, C. M., Oyedeji, A. O., Nxasana, N., Apalata, T., 
Antibacterial activity of the fruit of Napoleoneae imperialis, J. Innov. Res. Health Sc. Biotech., 2015, 1(1), 1-11. DOI: 10.18644/jiresh-biotech.0000001

14. Idu, M., Umweni, A., Odaro, T., Ojelede, L., Ethnobotanical plants used for oral healthcare among the Esan tribe of Edo state, Nigeria, Ethnobotanical Leaflets, 2009, 13, 548-563.

15. Jack, I. R., Ekong, R. E., Ndukwe, G. I., Phytochemical constituents of dichloromethane fraction and essential oil of Napoleonaea imperialis rind, Journal of Pharmacognosy and Phytochemistry, 2020, 9(5), 56-66. DOI: https://doi.org/10.22271/phyto.2020.v9. i5a.12664

16. Ojinnaka, C. M., Okpala, D. C., A Molluscicidal triterpenoid saponin from the fruits of Napoleonaea imperialis $\mathrm{P}$. Beauv. (Lecythidacaea), J. Appl. Sci. Environ. Manage., 2012, 16(2), 213216.

17. Ndukwe, G. I., Ojinnaka, C. M., Oyedeji, A. O., Novel bioactive triterpenoid saponin from the fruits of Napoleonaea imperialis P. Beauv. (Lecythidacaea), International Journal of Chemical Studies, 2016, 4(5), 80-87.

18. Jagessar, R., Phytochemical Screening and TLC profile of Montricardia arborescens, American Journal of Research Communication, 2017, 5(1), 129-142.

19. Mahato, B. S., Kundu, P. A., ${ }^{13}$ CNMR spectra of pentacyclic triterpenoids- a compilation and some salient features, Phytochemistry, 1994, 37(6), 1517-
1575.

20. Begum, S., Zehra, Q. S., Hassan, I. S., Siddiqui, S. B., Noroleanane triterpenoids from the aerial parts of Lantana camara, Helvetica Chimica Acta, 2008, 91(3), 460-467.

21. Ndukwe, G. I., Oluah, A., Fekarurhobo, G. K., Isolation of an isoflavonoid and a terpenoid from the heartwood of Baphia nitida Lodd. (camwood), Ovidius University Annals of Chemistry, 2020, 31(1), 5-8. DOI: 10.2478/auoc-20200002

22. Yin, F., Pan, R., Chen, R., Hu, L., Saikosaponins from Bupleurum chinense and inhibition of HBV DNA replication activity, Natural Product Communications, 2008, 3(2), 155-162.

23. Nose, M., Amagaya, S., Takeda, T., Ogihara, Y., New derivatives of saikosaponin, Chemical and Pharmaceutical Bulletin, 1989, 37(5), 1293-1296.

24. Sindambiwe, B. J., Balde, M. A., Bruyne, D. T., Pieters, L., Heuvel, V. H., Claeys, M., Triterpenoid saponins from Maesa lanceolata, Phytochemistry, 1996, 41(1), 269-277.

25. Ding, K., Guo, S., Rong, W., Li, Q., Liu, R., Xu, H., Yin, Y., Bi, K., A new oleanane-type pentacyclic triterpenoid saponin from the husks of Xanthoceras sorbifolim Bunge and its neuroprotection on $\mathrm{PC} 12$ cells injury induced by A $\beta 25-35$, Natural Product Research, 2019, 21(2), 1-7. 
26. Mendes, A. C. S., Mansoor, A. T., Rodrigues, A., Armas, B. J., Ferreira, U. M., Anti-inflammatory guaiane-type sesquiterpenes from the fruits of Pittosporum undulatum, Phytochemistry, 2013, 95, 308-314.

27. Parveen, A., Farooq, M. A., Kyunn, W. W., A new oleanane-type saponin from the aerial parts of Nigella sativa with antioxidant and anti-diabetic potential, Molecules, 2020, 25, 2171-2184.

28. Lunga, K. P., Qin, X., Yang, W. X., Kuiate, J., Du, Z., Gatsing, D., Antimicrobial steriodal saponin and oleanane-type triterpenoid saponins from Pallinia pinnata, Complementary and Alternative Medicine, 2014, 14, 369-376.
29. Navarro, P., Giner, M. R., Recio, C. M., Manez, S., Cerda-Nicolas, M., In-vivo inflammatory activity of saponins from Bupleurum rotundifolium, Life Sciences, 2001, 68(10), 119-1206.

30. Shital, A., Santosh, B., Rabindra, N., Invitro cytotoxic activity of novel oleanane type of triterpenoid saponin from stem bark of Manikara zapota Linn., Asian Journal of Pharmaceutical clinical Research, 2012, 5(4), 183-188.

31. Laszczyk, M. N., Pentacyclic triterpenes of the lupane, oleanane and ursane group as tools in cancer therapy, Planta Medica, 2009, 75(1), 1549-1560. 